Piotr Furtak, Michał Ceremuga and Jerzy Siczek

\title{
2. RADIATION DETECTION AND PROTECTION PROCEDURES
}

\subsection{Dosimetric equipment to measure the dose and dose rate/ equivalents of doses and equivalents dose rate of radiation}

Dose rate is the dose absorbed in unit time and indicates the amount of radioactive dose received by a person within a certain period of time. The dose rate is often given in thousandths of sieverts per hour. Depending on the calibration procedures used by the manufacturer, detectors provide information about the strength of the radioactive source.

\subsubsection{Operational dose quantities}

It is not possible to directly, and physically measure the amount of energy deposited (i.e. doses) in organs or people. Therefore alternative operational dose quantities are used by international convention to provide best and accepted estimates. It is Good Practice to measure doses to personnel with a personal dosimeter, but an alternative method is to estimate the effective dose with a hand held monitoring instrument.

The convention in radiation protection for area monitoring is to use:

Ambient dose equivalent - for control of effective dose to people, e.g. area monitoring. Ambient does equivalent $\mathrm{H}^{*}(\mathrm{~d})$ is the normal monitoring quantity for $\mathrm{X}$, gamma and neutron radiation where $\mathrm{d}$ is the depth at which the dose rate applies. International convention in radiation protection is to use the ambient dose equivalent at $10 \mathrm{~mm}$ depth i.e. $\mathrm{H}^{*}(10)$. The ambient dose gives a conservative estimate of the effective dose a person would receive when staying at the point of the monitoring instrument.

Directional dose equivalent - for control of doses to skin and lens of eye. Directional dose equivalent $\mathrm{H}^{\prime}(\mathrm{d})$ is intended for use with less penetrating radiation. Its main use is for skin dose at a depth of $0.07 \mathrm{~mm}$, but it is also used for dose to the eye lens at $3 \mathrm{~mm}$. Operational quantities that should be measured using portable instruments during dose rate surveys are therefore either:

- ambient dose equivalent at $10 \mathrm{~mm}$ depth, $\mathrm{H}^{*}(10)$ - SI unit: sievert (Sv);

- ambient dose equivalent rate, $\mathrm{H}^{*}(10) \mathrm{h}^{-1}$ - SI unit: sievert per hour $\left(\mathrm{Sv} \mathrm{h}^{-1}\right)$; 
- directional dose equivalent at $0.07 \mathrm{~mm}$ depth, $\mathrm{H}^{\prime}(0.07)$ - SI unit: sievert (Sv);

- directional dose equivalent rate, $\mathrm{H}^{\prime}(0.07) \mathrm{h}^{-1}$ - SI unit: sievert per hour $\left(\mathrm{Svh}^{-1}\right)$.

Some instruments may use different quantities such as fluence rate, air kerma rate, absorbed dose rate in air. Estimation of dose is a specialist task when using instruments not scaled in dose equivalent or dose equivalent rate.

\subsubsection{Measurement of contamination}

The units most commonly found on instruments used for contamination monitoring are:

- counts per second $\left(\mathrm{cs}^{-1}, \mathrm{cps}, \mathrm{ps}^{-1}\right.$, and $\left.\mathrm{s}^{-1}\right)$;

- disintegrations per minute (dpm);

- becquerel $(\mathrm{Bq})$;

- becquerels per square centimetre $\left(\mathrm{Bq} \mathrm{cm}^{-2}\right)$.

If the instrument indicates 'becquerel' or 'becquerel per square centimetre', a calibration factor will have been stored in the instrument. Conversion of counts per second to becquerels or becquerels per square centimetre can be complicated. This task requires a comprehensive knowledge of decay schemes, instrument performance and some estimation of how the local conditions (e.g. surface construction) might effect the observed count rate.

\subsubsection{Examples of dosimetric equipment to measure the dose and dose rate}

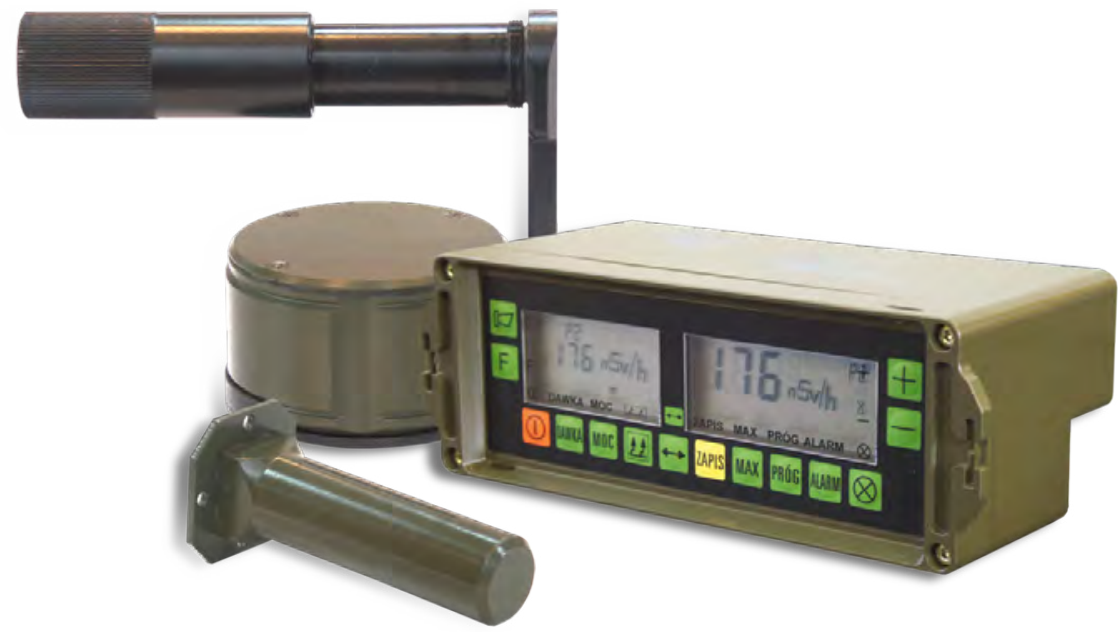

Figure 1. DPO radiometer 
DPO radiometer is designed for measurement of $\mathrm{X}$ and gamma radiation ambient dose equivalent rate and ambient dose equivalent. The device enables also detection and measurement of surface contamination with alpha, beta and gamma radiation emitters. The appliance can be installed not only in stationary military defensive and protective objects or command points but also can be treated as a primary dosimetry unit, used on ships and other buoyant objects of navy. It is also possible to use it as a a handheld radiometer in the field research.

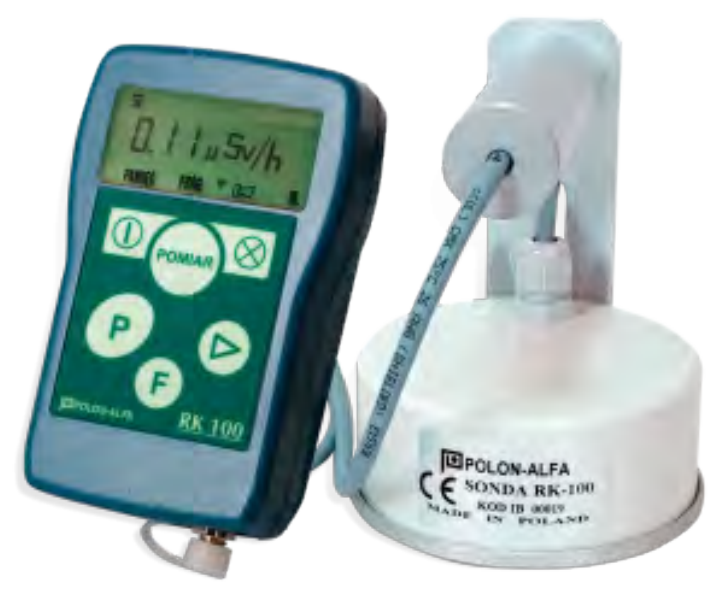

Figure 2. RK-100-2 radiometer

Portable radiometer RK-100-2 is dedicated for measurement of ambient dose equivalent rate, ambient dose equivalent, absorbed dose rate in air, absorbed dose in air of X, gamma radiation and surface contamination (alpha, beta and gamma emitters). It is easy to use radiometer with a durable case and light weight.

The SVG 2 is a newly developed, hand-held, hardened microprocessor controlled radiation detector based on state-of-the-art semiconductor technology. The SVG 2 consists of a base instrument with integrated energy-compensated MOSFET and PiN-diode sensors for neutron radiation detection, an external personal dosimeter which records the assimilated dose of the respective carrier, and an external probe. Additional probes, like a scintillation probe and a Geiger-MullerCounting Tube can be connected. The base instrument is easy to handle and is particularly distinguished by its low weight and power consumption. Not only the curent dose rate but also the integrated dose over a certain time can be displayed. Additionally, the initial and assimilated dose of radiation is measured by an internal dosimeter, even if the instrument is shut off! Both modes of total and differential dose display are available. Alarm thresholds for all the modes are adjustable. The alarm is given either optically by a red flashlight and acoustically by a horn $(>90 \mathrm{~dB}$ A) or only by the flashlight. An earphone can alternatively be used. 


\subsubsection{Spectrometric equipment for identification of the radioactive sources}

The devices listed in this section are able to identify radioactive isotopes by analyzing the characteristic gamma emission spectra. The devices are often multipurpose/multitask instruments able to detect different forms of radiation and perform a variety of functions; used to search, detect and identify radioactive materials.

Hand-held Radioisotope Identification Devices (RIIDs) take this another step further and are designed to identify the isotopic composition of radioactive sources. A RIID is typically a small, handheld device that is generally easy to use and deploy. The devices are often multipurpose/multitask instruments able to detect different forms of radiation and perform a variety of functions; used to search, detect and identify radioactive materials. Radioisotope Identification Devices (RIIDs) are instruments that are designed to determine the identity of radioactive materials by measuring the energy of the emitted gamma rays. Law enforcement, customs, and other personnel are being equipped with RIIDs as part of a national strategy to interdict illicit movement of radioactive material. When radiation sources are detected by screening devices such as radiation portal monitors or radiation pagers, RIIDs are used to determine whether the source of radioactivity constitutes a high level threat. Radiological emergency personnel, firefighters and other response personnel also use RIIDs for situational assessment during radiological emergencies.

Most radioisotopes emit gamma rays with characteristic energies. Gamma rays emitted by a radioactive source strike a detector within the RIID and are converted Into a signal that indicates the energy of the incident gamma ray. The number of gamma rays at each energy are counted and plotted versus energy in an energy spectrum which reveals characteristic energy peaks. Identification is based on matching the peaks in the spectrum to the known peaks and peak ratios of gamma emitters. This matching process is done using proprietary isotope identification software which is a critical component of these instruments. Two key RIID features are energy resolution and sensitivity.

Resolution is a measure of how close two energy peaks can be and still be differentiated; the lower the percent resolution, the better the detectors' ability to distinguish two or more closely spaced peaks.

Sensitivity is a measure of how efficiently incoming gamma rays are detected; this determines the counting time needed to obtain a spectrum

Two different types of detectors are used in commercially available RIIDs.

1. Scintillator detectors are transparent materials (crystals) that, when struck by gamma rays, produce light pulses with intensities that are proportional to the gamma ray energies. These light pulses are converted to electrical pulses by a photomultiplier tube (PMT) and then processed by a multi-channel 
spectrometer to produce characteristic gamma ray spectra. RIIDs using scintillation detectors are lighter and less expensive than other technologies, but have poorer resolution. The most common scintillator material used in RIIDs is thallium doped sodium iodide $(\mathrm{NaI}(\mathrm{Tl}))$ crystals. A relatively new scintillator material, cerium doped lanthanum bromide $(\mathrm{LaBr} 3: \mathrm{Ce})$ has twice the resolution of $\mathrm{NaI}(\mathrm{Tl})$, and also has a higher light output, but RIIDs using this material could cost about twice as much.

2. Semiconductor detectors are made from specially processed crystalline material. Gamma rays striking the detector create free positive and negative charges that are detected as a current; the higher the energy of the gamma ray, the higher the current that is generated. The most common semiconductor material used in RIIDs is high purity germanium (HPGe) crystals ( 2 inches dia.x 1.2 inches high), these must be cooled to liquid nitrogen temperatures $\left(-321^{\circ} \mathrm{F}\right)$ with a built-in refrigeration system in order to operate. HPGe RIIDS are much larger and heavier, and cost about fivetimes as much as $\mathrm{NaI}(\mathrm{Tl})$ RIIDS. While scintillator RIIDs are ready to operate in a few minutes, an HPGe based RIID that is at room temperature is inoperable until it is fully cooled, which takes approximately two hours.

Handheld RIIDs are battery powered, have built- in software for spectral analysis and are capable of identifying the radioisotopes most commonly encountered by emergency responders. Radioactive isotopes are divided into four groups:

- special nuclear material (SNM), plutonium, highly enriched uranium;

- medical isotopes (used in radiotherapy and medical imaging;

- industrial isotopes (used in weld inspection devices, civil engineering equipment, food irradiators);

- naturally occurring radioactive material (NORM), commercial products such as ceramics and fertilizers containing radioactive elements such as potassium, uranium, thorium and radium.

\section{Limitations}

Radioactive sources can be shielded so that gamma or neutron radiation is below the RIID detection limits or the spectrum is greatly distorted. It is particularly easy to shield SNM isotopes that emit only low energy gamma rays. For example, about 1 inch of lead will reduce the emission from plutonium by about a factor of 1000, and 0.25 inch of lead will reduce the emission from HEU by the same factor. The energy calibration of some RIIDs can be affected by temperature changes such as moving between indoors and outdoors, which may result in misidentifications. Some devices contain a built in check source for recalibration. Isotope identification software currently used in RIIDs can misidentify radioactive sources even when the data was collected with a properly 
calibrated RIID. For this reason, the DHS Domestic Nuclear Detection Office established the Joint Analysis Center (JAC) Regional Reachback Program. Highly trained gamma spectrometry specialists are available on a $24 / 7$ basis through this program to provide analysis of RIID data to first responders.

\section{Example of spectrometric equipment for identification of the radioactive sources}

The identiFINDER R500 (radHUNTER) is an extremely sensitive and accurate digital hand-held gamma radionuclide identification device (RID). It is the culmination of over eight years of development of micro-miniature, digital signal processing electronics; operating power conservation; and advancements in the scintillation detector, radionuclide template matching identification algorithm. The identiFINDER R500 development was supported in part by the U.S. Department of Homeland Security (DHS) / Domestic Nuclear Detection Office (DNDO), Human Portable Radiation Detection System (HPRDS) program.

Available in two configurations ( $\mathrm{NaI}$ and $\mathrm{LaBr}$ ), the identiFINDER R500 is able to quickly detect, rapidly locate, accurately measure and precisely identify sources of contamination from their gamma radiation signature. The identiFINDER R500 NGH ultra 3n uses a 0.75 " thick by 4 " diameter $\mathrm{NaI}(\mathrm{Tl})$ detector while the identiFINDER R500 LGH ultra 3 n uses a 1.5 " by 1.5 " LaBr3:(Ce) detector. Both instruments come with a GM tube for high gamma dose rate measurements and a sealed $\mathrm{He} 3$ detector with moderator for neutron detection. Each instrument is supplied in a carrying case with recharging unit, communication cables, synchronization and analysis software. Variations in normal operating conditions can affect the performance of RID with scintillation detectors such as $\mathrm{NaI}(\mathrm{Tl})$ and $\mathrm{LaBr} 3:(\mathrm{Ce})$. Dramatic temperature variations can affect the scintillation detector and/or the PMT and can also cause instabilities and non-linearities in the electronics.

The InSpector ${ }^{\mathrm{rx}} 1000$ is a high-performance, hand-held $\mathrm{NaI}$ spectrometer for use primarily in first responder, customs, homeland security, and health-physics applications. One-click simplicity masks the sophisticated spectral processing facilities that lie within the instrument-providing alevel of performance previously available only in more complicated laboratory systems. The InSpector 1000 is an instrument that provides answers not just data! With the InSpector 1000, users from law enforcement officers to Health Physics technicians can obtain the results they desire with an easy to use, intuitive interface. InSpector 1000 has been designed for easy operation. The high-resolution color LCD display is clearly visible from bright sunlight to night conditions. The unit can be held and operated in one hand (even if wearing gloves). The convenient hand strap leaves the hand positioned to easily reach the controls with the thumb. This leaves the 
second hand free for other operations - holding a ladder, sorting through suit cases or packages, etc. In addition, for applications requiring flexible positioning of the detector relative to the object being inspected, the detector can be detached from the instrument body and placed in any position or narrow gap. This greatly increases the probability of detecting small amounts of radiation and precisely locating sources by reducing the measurement distance.

InSpector 1000 was designed to be used in all types of environmental conditions. Its ruggedized, light weight packaging meets the IP 54 specifications and is easily decontaminated. It also has a wide operating range for both temperature and humidity. The InSpector 1000 instrument has been designed to survive a one meter drop onto concrete.

InSpector 1000 is an easy-to-use digital multichannel analyzer, ideally suited for homeland security applications, custom and border protection, healthphysics, treaty and non-proliferation compliance, monitoring of nuclear transportation, and in situ measurement of objects, surfaces etc. The user interface provides the ultimate flexibility in field operations. The InSpector 1000 is readily usable by less sophisticated users without the need of extensive training and also offers highlevel spectrometry analysis capabilities for expert users. It can be used in any field measurement application requiring nuclide identification, activity measurements, dose/count rate measurements, or spectrum acquisition and analysis. Although the instrument has a powerful suite of spectroscopy functions, the InSpector 1000 's simple user interface isolates the user from complicated spectrum analysis while still providing this data to more advanced users.

\section{References}

Radiation Detection and Measurement - G. Knoll.

http://www.canberra.com

www.mirion.com/

www.paa.gov.pl

www.polon-alfa-pl.

www.polon-ekolab.com.pl 